\title{
INVESTIGATING THE SUITABILITY OF MIRRORLESS CAMERAS IN TERRESTRIAL PHOTOGRAMMETRIC APPLICATIONS
}

\author{
A. H. Incekara ${ }^{a}, *$, D. Z. Seker ${ }^{b}$, A. Delen ${ }^{\text {a }}$, A. Acar ${ }^{\text {a }}$ \\ ${ }^{a}$ Gaziosmanpasa University, Department of Geomatics Engineering, Tokat, Turkey - (abdullah.incekara, ahmet.delen, \\ alparslan.acar)@gop.edu.tr \\ b Istanbul Technical University, Department of Geomatics Engineering, Istanbul, Turkey - seker@itu.edu.tr
}

KEY WORDS: Accuracy Assessment, Mirrored Camera, Mirrorless Camera, Rock Surface, Terrestrial Photogrammetry

\begin{abstract}
:
Digital single-lens reflex cameras (DSLR) which are commonly referred as mirrored cameras are preferred for terrestrial photogrammetric applications such as documentation of cultural heritage, archaeological excavations and industrial measurements. Recently, digital cameras which are called as mirrorless systems that can be used with different lens combinations have become available for using similar applications. The main difference between these two camera types is the presence of the mirror mechanism which means that the incoming beam towards the lens is different in the way it reaches the sensor. In this study, two different digital cameras, one with a mirror (Nikon D700) and the other without a mirror (Sony a6000), were used to apply close range photogrammetric application on the rock surface at Istanbul Technical University (ITU) Ayazaga Campus. Accuracy of the 3D models created by means of photographs taken with both cameras were compared with each other using difference values between field and model coordinates which were obtained after the alignment of the photographs. In addition, cross sections were created on the 3D models for both data source and maximum area difference between them is quite small because they are almost overlapping. The mirrored camera has become more consistent in itself with respect to the change of model coordinates for models created with photographs taken at different times, with almost the same ground sample distance. As a result, it has been determined that mirrorless cameras and point cloud produced using photographs obtained from these cameras can be used for terrestrial photogrammetric studies.
\end{abstract}

\section{INTRODUCTION}

Visualization of objects with the help of 3D models is one of the most effective way to demonstrate the current state of the modelled object so that several analyses can be made on model which has detailed informations regarding coordinates and texture (Avsar et al., 2008). The smallest component of a 3D model is point data which is one of the most preferred data types in diverse engineering projects and applications, especially when used in cloud form and digital photograhps obtained by means of digital cameras are basic data source in order to generate point cloud.

From past to present, different types of non-metric cameras are used in terrestrial photogrammetric applications such as cultural heritage (Frastia, 2005), architectural restoration and archaeological surveys (Jauregui and Jauregui, 2000), industrial measurements (Luhmann, 2010) or more specific topics like glacier monitoring (Kaufmann and Ladstadter, 2008). For these applications, digital single-lens reflex cameras (DSLR) which are commonly referred as mirrored cameras are generally preferred. Recently, digital cameras which are called as mirrorless systems that can be used with different lens combinations have become available for using similar applications. The compact dimensions of mirrorless cameras relative to mirrored ones are remarkable and the main difference between these two camera types is the presence of the mirror mechanism which means that the incoming beam towards the lens is different in the way it reaches the sensor as shown in Figure 1.

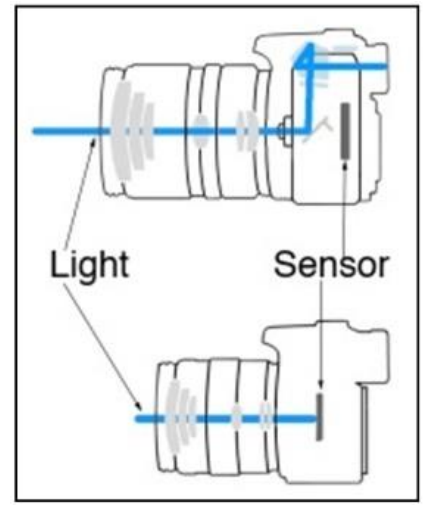

Figure 1. (C) Working mechanism of mirrored (upper) and mirrorless (bottom) cameras (Url-1, 2017)

For mirrored camera, light passing through the lens hits the mirror which is located in front of the sensor and goes from here to the pentaprism which is a special component that allows the view to look straight. Thereafter, light reaches the optical viewfinder. At the moment of shooting, the mirror lifts and the light falls into sensor (Url-2, 2017). There is no mirror mechanism in the mirrorless camera. Therefore, the light passing through the lens directly reaches the sensor. In mirrorless cameras, electronic viewfinder is used instead of optical one. Optical viewfinder transmits the frame seen by the camera to the eye with the help of objective and prisms. However, electronic viewfinder transmits the photograph to be taken by the camera to

\footnotetext{
* Corresponding author
} 
the eye, not the frame seen by the machine. The most important error source for optical viewfinder is parallax but it is more valuable thanks to real view and correct colors. Light, screen resolution and screen structure have more impact on electronic one. The detailed comparison for mirrored and mirrorless cameras is given in Table 1.

\begin{tabular}{|c|c|c|}
\hline Specification & Mirrored & Mirrorless \\
\hline Weight and Size & & $\checkmark$ \\
Lens Diversity & $\checkmark$ & \\
Viewfinder & $\checkmark$ & \\
Continuous Shutter Speed & & $\checkmark$ \\
Photograph Quality & 0 & 0 \\
Battery & $\checkmark$ & \\
Price & 0 & o \\
\hline \multicolumn{2}{|c}{} \\
\hline \multicolumn{2}{|c}{ Better }
\end{tabular}

Table 1. Comparison of mirrored and mirrorless cameras

Figure 2 represents the comparison of these cameras in terms of their dimensions. The presence of the mirror mechanism causes the DSLR cameras to have a larger body, thus heavier batteries are used for that type of body. On the contrary, in mirrorless cameras, the absence of the mirror mechanism results in a smaller body and less weight. This is one of the most important reasons why mirrorless cameras are also used in aerial photogrammetric applications performed by means of unmanned aerial vehicles. With lower weight, longer flight times and quality photographs as well as photographs obtained with the mirrored camera along with the appropriate lens combination make them more preferable.

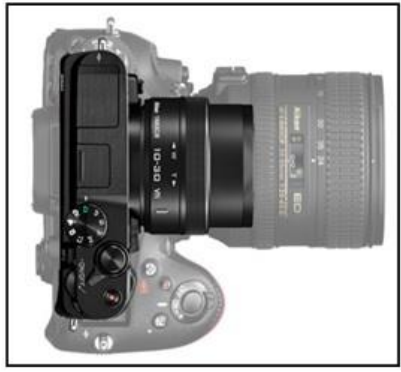

Figure 2. (C) Comparison of mirrored and mirrorless cameras in terms of dimensions (Url-3,2017)

\section{STUDY AREA}

A rock surface within Ayazaga Campus of Istanbul Technical University showed in Figure 3 was selected as study object in order to compare mirrored and mirrorless cameras with each other. The rock surface selected as study object is approximately $1 \mathrm{~m} \times 3 \mathrm{~m}$ in size. Preferred cameras are Nikon D700 which has mirror and Sony a6000 which has no mirror.

Changing of interior orientation elements in course of time is possible for non-metric cameras after certain period of exposure (Jechev, 2004) and calibration process has to be applied to control their stability and fix them if necessary (Rodríguez et al., 2008). After the calibration process, principal distance of Nikon D700 and Sony a6000 were determined as respectively $52.54 \mathrm{~mm}$ (standard value for the lens used is $50 \mathrm{~mm}$ ) and $16.02 \mathrm{~mm}$ (standard value for the lens used is $16 \mathrm{~mm}$ ). Mirrored Nikon D700 has been used for a long time with a lens with fixed focal length of $50 \mathrm{~mm}$.

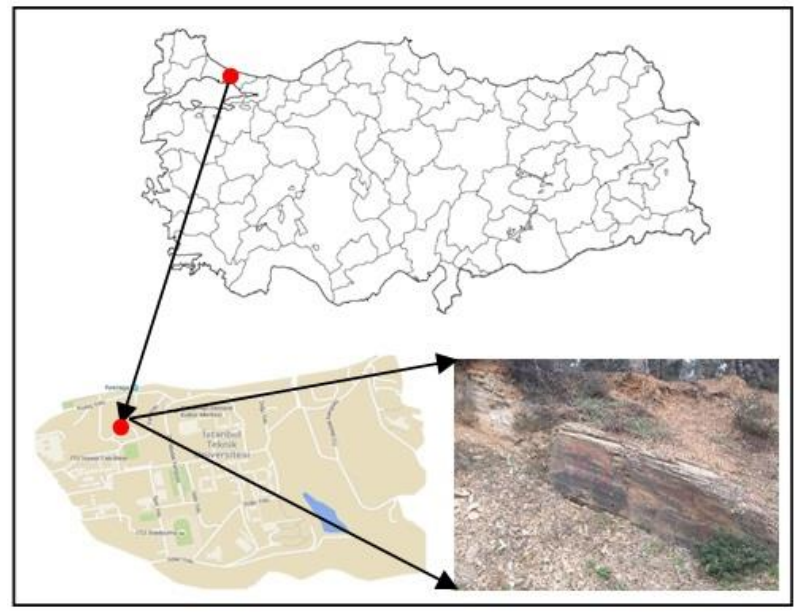

Figure 3. Study area

\section{ANALYSES}

3D object models were created with the integration of terrestrial geodetic measurements and terrestrial photographs. During the work, 17 control points were used. With both cameras, the photographs were taken in such a way that the pixel dimensions would be equal to each other. Process sequence of generating 3D models is obtaining weak point cloud after the alignment of the photographs, obtaining the dense point cloud after the densification of weak point cloud, generating mesh model over dense point cloud, covering mesh model with photographs taken and matching the model with control points measured by means of total station. Produced 3D models are given in Figure 4a and Figure 4b.

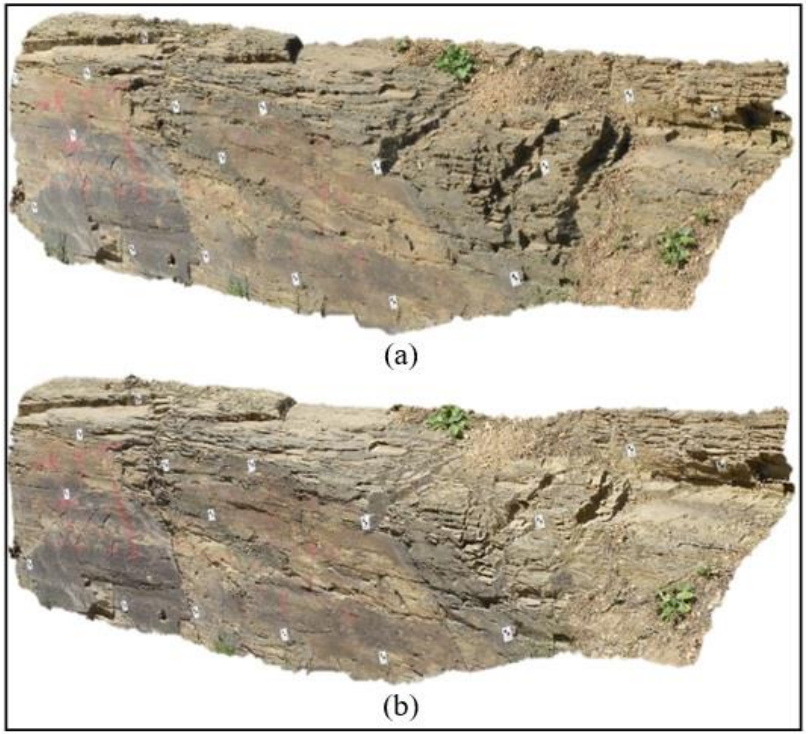

Figure 4. 3D object models produced by photographs taken by Nikon D700 (a) and Sony a6000 (b)

Accuracy analyses for 3D models were made by using difference values between field coordinates which are accepted as raw values measured in the field and model coordinates which are assumed as correct values after the generation. Accuracy results in the direction of 3 axis and model error which calculated by taking the square roots of the squares of these values are given in Tables $2 \mathrm{a}$ and $2 \mathrm{~b}$. 


\begin{tabular}{|c|c|c|c|}
\hline $\mathrm{m}_{\mathrm{x}}(\mathrm{mm})$ & $\mathrm{m}_{\mathrm{y}}(\mathrm{mm})$ & $\mathrm{m}_{\mathrm{z}}(\mathrm{mm})$ & $\mathrm{m}_{\text {model }}(\mathrm{mm})$ \\
\hline 2,3 & 3,9 & 0,8 & 4,6 \\
\hline
\end{tabular}

Table 2a. Accuracy analysis of 3D object model (Mirrored Nikon D700)

\begin{tabular}{|c|c|c|c|}
\hline $\mathrm{m}_{\mathrm{x}}(\mathrm{mm})$ & $\mathrm{m}_{\mathrm{y}(\mathrm{mm})}$ & $\mathrm{m}_{\mathrm{z}(\mathrm{mm})}$ & $\mathrm{m}_{\text {model }(\mathrm{mm})}$ \\
\hline 2,1 & 4,4 & 1,2 & 5,0 \\
\hline
\end{tabular}

Table 2b. Accuracy analysis of 3D object model (Mirrorless Sony a6000)

Photographs were taken again three times with the same distances (values calculated separately for both cameras) at different times with both cameras and 3D object models were created all over. The distance used in the first photo shooting with the mirrored camera is the same as the distance used in the other shooting processes. This situation is also valid for mirrorless camera. That is, the distance to take photographs for mirrored and mirrorless camera is not the same. Otherwise, the consistency of the cameras could not be compared because the GSD values would be different. In this step, it was examined consistency of model coordinates within themselves. In accordance with this, the coordinates of the control points of the other three models that were produced later on were compared with those of the first produced models and maximum changes in the $\mathrm{x}, \mathrm{y}$, and $\mathrm{z}$ directions were determined as shown in Table $3 \mathrm{a}$ and $3 \mathrm{~b}$.

\begin{tabular}{|c|c|c|}
\hline $\operatorname{Max} \Delta \mathrm{x}(\mathrm{mm})$ & $\operatorname{Max} \Delta \mathrm{y}(\mathrm{mm})$ & $\operatorname{Max} \Delta \mathrm{z}(\mathrm{mm})$ \\
\hline 0,8 & 1,2 & 1,1 \\
\hline
\end{tabular}

Table 3a. Coordinate change for other models according to the first model produced (Mirrored Nikon D700)

\begin{tabular}{|c|c|c|}
\hline $\operatorname{Max} \Delta \mathrm{x}(\mathrm{mm})$ & $\operatorname{Max} \Delta \mathrm{y}(\mathrm{mm})$ & $\operatorname{Max} \Delta \mathrm{z}(\mathrm{mm})$ \\
\hline 1,7 & 4,9 & 2,6 \\
\hline
\end{tabular}

Table $3 \mathrm{~b}$. Coordinate change for other models according to the first model produced (Mirrorless Sony a6000)

Cross sections were created on the 3D models (Figure 5) for both data source and maximum area difference between them were analyzed (Table 4). The start and end points of the cross sections and other places where they pass along their direction are the middle part of the control points, that is the part measured in the field. Figure 6 represents a part of the cross section in the direction of control points number 2-4-6-8-10 and maximum area difference between both data source.

\section{RESULTS AND CONCLUSIONS}

Two cameras were used on the rock surfaces, one with mirror and the other without mirror, to investigate the usability of mirrorless cameras for terrestrial photogrammetric applications.

According to measurements done in the field and analyses carried out afterward;

-Very close values were obtained in the analysis of accuracy.

-No significant change has been observed in the accuracy analysis of the control points with different numbers and different distributions from each other.

-The mirrored camera gave more consistent results within itself in the coordinate changes with respect to different photographs taken at the same distance.

-The sections created on the model are also almost overlapping, and the maximum area difference values between the sections are quite small. This also means that any study to be made on rock surfaces such as determining the roughness angles of rock surfaces via cross sections will be almost the same.

-The reason why $\Delta \mathrm{y}$ values are higher than $\Delta \mathrm{x}$ and $\Delta \mathrm{z}$ and similarly $m_{y}$ values are higher than the $m_{x}$ and $m_{z}$ is that the horizontal distance between two polygon points which were used for measurement of control points marked on the surface is the coordinate difference in the direction of $\mathrm{Y}$ axis.

- The full frame feature and optical viewfinder of the used mirrored camera and the use of a fixed focus lens make it possible to take pictures closer to reality. The mirrorless camera used is not full frame and the lens used is not fixed focus. If a fixed focus lens is used for mirrorless camera, the number of moving parts in the camera at the time of shooting will be fewer and better photographs will be obtained.

-That the cameras are alternative to each other can be demonstrated by using them for more detailed studies of rock surface characterization. Similar studies should be performed using different objects in order to make better comparisons.

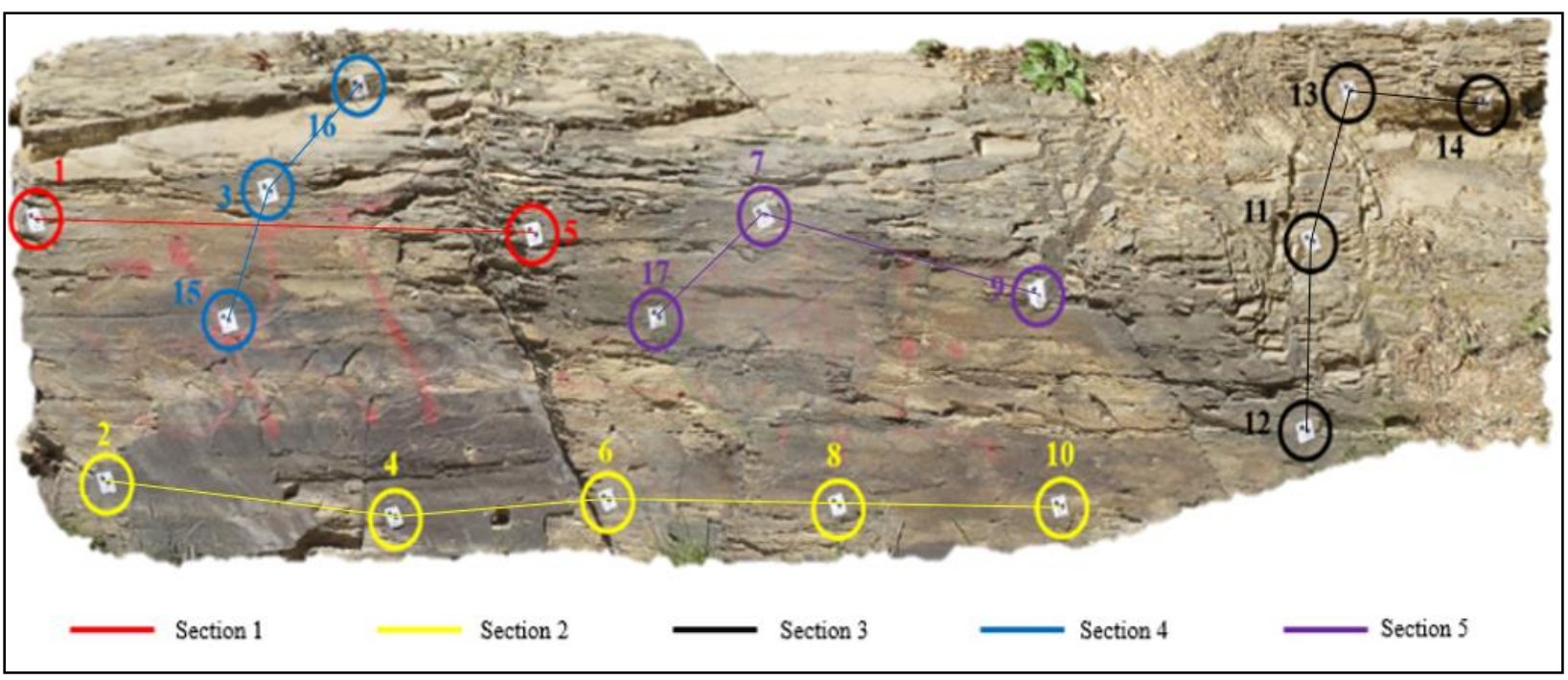

Figure 5. Points where five cross-sections pass 


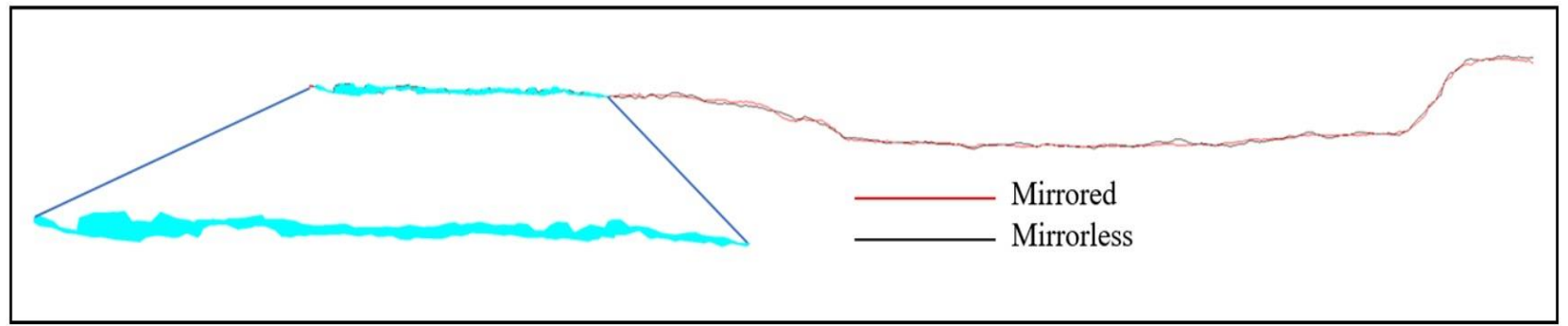

Figure 6. Section 2 of mirrored Nikon D700 and mirrorless Sony a6000 in the direction of control points 2-4-6-8-10 and maximum area difference between them

\begin{tabular}{|c|c|c|}
\hline $\begin{array}{c}\text { Cross Section } \\
\text { No }\end{array}$ & $\begin{array}{c}\text { Cross Section } \\
\text { Direction }\end{array}$ & $\begin{array}{c}\text { Maximum Area } \\
\text { Difference }\left(\mathrm{m}^{2}\right)\end{array}$ \\
\hline 1 & $1-5$ & 0.0002 \\
2 & $2-4-6-8-10$ & 0.0009 \\
3 & $12-11-13-14$ & 0.0006 \\
4 & $16-3-15$ & 0.0001 \\
5 & $17-7-9$ & 0.0003 \\
\hline
\end{tabular}

Table 4. Comparison of the cross sections

\section{REFERENCES}

Avsar, E.Ö., Duran, Z., Akyol, O., Toz., G (2008). Modelling of the Temple of APOLLO SMINTHEUS using photogrammetry and virtual reality, The International Archives of the Photogrammetry, Remote Sensing and Spatial Information Sciences. Vol. XXXVII. Part B5. Beijing 2008

Frastia, M. (2005). Possibilities of using inexpensive digital cameras in applications of close range photogrammetry, Slovak Journal of Civil Engineering, 2005/2, pp. 20-28.

Jauregui, L.M., \& Jauregui, M. (2000). Terrestrial photogrammetry applied to architectural restoration and archaeological surveys, International Archives of Photogrammetry and Remote Sensing, Vol. XXXIII, Part B5, Amsterdam 2000.

Jechev, D. (2004). Close range photogrammetry with amateur camera, XXth ISPRS Congress, Proceedings of the Youth Forum (ISPRS 2004), pp. 136-138.

Kaufmann, V., \& Ladstadter, R. (2008). Application of terrestrial photogrammetry for glacier monitoring in Alpine environments, Proceedings of the $21^{\text {st }}$ Congress of ISPRS, Beijing, 3-11 July, Vol. 37, Part B8, pp. 813-818.

Luhmann, T. (2010). Close range photogrammetry for industrial applications, ISPRS Journal of Photogrammetry and Remote Sensing, 65, pp. 558-569.

Rodríguez, B.D.D, García, S.H.G., \& Piñero, Y.B (2008). Experience using non-metric cameras in photogrammetry, International Archives of the Photogrammetry, Remote Sensing and Spatial Information Sciences, vol. XXXVII, part B5, pp. 769-772.

Url-1: https://www.techly.com.au/2014/01/04/mirrorlesscameras-rip-dslrs-still-need-mirror-ces-update/ (2 Aug 2017)
Url-2: https://www1.crutchfield.com/learn/compact-systemcamera-buying-guide.html (19 Apr 2017)

Url-3: http://www.senelfoto.com/dslr-aynasiz/ (19 Apr 2017) 